\title{
Dynamics and spatial genomics of the nascent transcriptome in single mESCs by intron seqFISH
}

Sheel Shah ${ }^{* 1,2}$, Yodai Takei*1, Wen Zhou* ${ }^{* 1}$, Eric Lubeck ${ }^{3}$, Jina Yun ${ }^{1}$, Noushin Koulena ${ }^{1}$, Eric J. Liaw ${ }^{2}$, Mina $\mathrm{Amin}^{4}$, and Long $\mathrm{Cai}^{1}$

1. Division of Biology and Biological Engineering, Caltech, Pasadena USA 91125

2. UCLA-Caltech Medical Scientist Training Program, David Geffen School of Medicine, University of California at Los Angeles, Los Angeles, CA 90095, USA

3. Current Address: Department of Bioengineering, Stanford University, Stanford, CA 94305, USA

4. Current Address: UC Riverside School of Medicine, University of Riverside, Riverside, USA 92521

* these authors contributed equally.

Correspondence should be addressed to Icai@caltech.edu

\section{Summary.}

Recent single cell experiments have revealed significant heterogeneities at the levels of transcription, DNA methylation and chromosome organization in individual cells. However, existing method of profiling mRNAs effectively averages transcriptional dynamics over many hours due to hours-long life time of most mRNAs. To capture the instantaneous activity of the transcriptome that reflects the rapid regulatory changes in cells, we imaged up to 10,421 nascent transcription active sites (TAS) in single mouse embryonic stem cells using seqFISH followed by multiple rounds of single molecule FISH and immunofluorescence. We observed that nascent transcription active sites appear to be distributed on the surface of individual chromosome territories and are dispersed throughout the nucleus. In addition, there are significant variability in the number of active transcription sites in single cells, representing globally more active to quiescent states. These states interconverted on the time scale of 2 hours as determined by a single cell pulse-chase experiment. Thus, transcriptome level seqFISH experiments provide an unprecedented spatial and dynamic view of chromosome organization and global nascent transcription activity in single cells.

\section{Introduction}

Recent single cell experiments in mouse embryonic stem cells (mESCs) revealed heterogeneity at the transcriptional and protein levels (Chambers et al., 2007; Hayashi et al., 2008; Toyooka et al., 2008; Kumar et al., 2014; Klein et al., 2015; Kolodziejczyk et al., 2015), chromosome structures (Stevens et al., 2017) as well as epigenetic states (Hayashi et al., 2008; Marks et al., 2012; Singer et al., 2014;

Smallwood et al., 2015; Angermueller et al., 2016). However, because most mRNAs have half-life of several hours in mammalian cells, mRNA levels average expression dynamics over many hours and obscure potential rapid changes in global transcriptional activity.

We set out to profile the global instantaneous transcriptional activity and chromosome structure of 
individual mESCs cells by Fluorescence in situ hybridization (FISH). We reason that instantaneous transcriptional profiling could reveal phenomenon occurring on a faster time scale compared to the longer-lived mature mRNA. In addition, FISH approaches can directly image gene loci to reveal the spatial organization of chromosomes in single nuclei.

Pioneering work with single molecule FISH (smFISH) (Femino et al., 1998; Raj et al., 2006) observed that nascent mRNAs are produced in bursts at transcription active sites (TAS) in individual nuclei. In particular, these nascent sites of transcription near the genomic loci can be selectively labeled over mature transcripts, by targeting introns, which are co-transcriptionally processed out (Levesque and Raj, 2013). This intron chromosomal expression FISH (iceFISH) assay (Levesque and Raj, 2013) showed that at least 20 TAS from a single chromosome can be detected to measure their spatial position and expression levels in single human cells.

Here, we applied the sequential FISH (seqFISH) strategy (Lubeck et al., 2014) to extend the iceFISH approach to the transcriptome level. seqFISH has been used to image hundreds of mRNAs in tissues and revealed distinct spatial structures in the mouse brain (Shah et al., 2016). The multiplex capacity of seqFISH is only limited by the density of the RNAs in cells and optical diffraction limit. Because nascent TAS occur infrequently and appear only near their genomic position in the nuclei, it is possible to resolve nascent site of synthesis across the genome in the same nucleus without optical crowding. Furthermore, each TAS often consists of multiple nascent transcripts, enhancing the signal detected.

Using intron seqFISH, we profiled the instantaneous transcriptional activity of up to 10,421 genes in single mESCs, along with mRNA, long noncoding (Inc)RNA seqFISH and immunofluorescence to detect the pluripotency markers, cell cycle markers and nuclear bodies in the same single cells. We observed that active transcription occurs at the surface of chromosome territories and are distributed throughout the nucleus. We also observed that there are significant variability in the global transcriptional activities in individual cells. We showed that global instantaneous transcription activities are fluctuating rapidly on a 2 hour time scale using a single cell pulse chase experiment. The imaging approach taken here provides new information on spatial organization of loci and gene expression dynamics in single cells.

\section{Results.}

\section{Intron seqFISH targets transcriptionally active loci in single cells.}

To multiplex thousands of TAS loci, we used sequential rounds of hybridization to generate a unique temporal barcode sequence on each TAS, which can then be decoded by aligning images from each round of hybridization (Figure 1A-1B). Specifically, we target the introns at the $5^{\prime}$ regions of genes by a set of primary probes, which contain an overhang sequence that can be hybridized by the readout oligos that are labeled with fluorophores (Figure 1C). The cells are imaged on a spinning disk confocal microscope with z-sections. Then the readout probes are removed by denaturation in $70 \%$ formamide, while the primary probes remain bound on the intronic RNA due to longer probe size and higher DNARNA affinity. A different set of readout probes is then hybridized to the primary probes that switches the dye labels on each of the loci. Repeating the imaging, stripping and rehybridization $\mathrm{N}$ times provides $\mathrm{F}^{\mathrm{N}}$ barcodes, where $\mathrm{F}$ is the number of distinct fluorophores. With one additional round for error correction (Shah et al., 2016), we can correct for loss of signal in any round of hybridization due to mishybridization (Figure 1A). 
We first targeted the introns of 1,000 transcription factors that are conserved between mouse and human (Fulton et al., 2009) and are distributed across all of the chromosomes with 48 probes per gene. 6 rounds of hybridization and 4 fluorophores $\left(4^{6}=4096\right)$ can decode all 1,000 genes with one round of error correction. We observe negligible false positive rates in the decoding with the error correction scheme used (Figure 1B) with an average $188 \pm 108$ (mean \pm standard deviation) on-target barcodes detected per celland $2.8 \pm 2.3$ (mean \pm standard deviation) off-target barcodes detected per cell (Figure 1D). In individual cells, the number of active sites from each gene ranged from 0 to 4 per cell (Figure $1 E$ ), as some cells are in $\mathrm{G} 2 / \mathrm{M}$ phase and contain 4 chromosomes. The estimated detection rate is $81 \%$ by comparing the number of dots detected in the first round of hybridization and the number of barcodes decoded. Compared to bulk GRO-seq data (Jonkers et al., 2014), which measures the amount of productively elongating RNA polymerase II and reflects the nascent transcriptional activity of genes, we observed a correlation of 0.57 with the average burst frequency of each gene in the seqFISH decoded data set. This comparison indicates the overall agreement between the burst frequency of active loci measured directly by intron seqFISH and the density of polymerases on gene loci measured by GRO-seq.

\section{Active transcription occurs on the surface of chromosomes territories.}

We next used the 1,000 gene intron seqFISH data to characterize the spatial organization of TAS in single $\mathrm{mESCs}$. The data revealed that spatial distribution of TAS appears uniform across the nucleus, excluded from the DAPI dense heterochromatic regions as well as from the nucleoli (Figure 2A). There does not appear to be major factories of active transcription in the nucleus, although local foci cannot be ruled out. Reconstruction of the intron seqFISH data revealed that TAS from each chromosome appeared to span spatial territories (Figure 2A, right panel), consistent with previous observations (Mahy et al., 2002a; Bolzer et al., 2005) of chromosome territories (CTs) by chromosome paint experiments.

How are the TAS organized with respect to the CTs? To determine the relative positioning of the TAS and the CTs, we combined intron FISH targeting the TAS and chromosome paint probe hybridization to directly visualize the individual CTs. We observed that TAS are located on the surfaces of CTs (Figures 2B-D). We also found that occasionally some TAS can be located a significant distance away from the core CTs (Figure 2C-D). On average, TAS are located $0.65 \pm 0.82 \mu \mathrm{m}$ (mean \pm standard deviation) exterior relative to their $\mathrm{CT}$ edge (Figure $2 \mathrm{C}$ ). This is consistent with previous observations that regions containing coding sequences are positioned away from rest of the chromosome (Mahy et al., 2002b; Boyle et al., 2011). We found this feature is consistently observed across all profiled chromosomes, suggesting that surface RNA transcription is a universal feature of mESC chromosome. Several genes, such as Pten, Ppp3r1 and Ctnna1, showed lower intrachromosomal contacts compared to other genes, suggesting they are looped away from the main chromosome territory (Figure 2D). Individual intron FISH against these genes confirmed that they frequently loop out from their chromosomes and appear either on the surfaces of nucleoli or along the nuclear periphery (Figure 2D).

Next, we asked whether genes are dynamically localized to the surface of CTs depending on their activity. By performing intron FISH, DNA FISH and chromosome paint in sequential steps in the same cell (Figure 2E), we measured the spatial positioning of loci relative to the CT surface, as well as their instantaneous transcriptional activities. Notably, for the 9 genes we investigated, spatial positionings of loci were not influenced by their instantaneous transcriptional activities (Figure 2F). 
We also asked how the TAS from different sets of chromosomes interacted with one another. As most of the TAS are present at the surface of the CTs or looped away from their core CTs, we observed many interchromosomal contacts and fewer intrachromosomal contacts. These interchromosomal contacts are highly variable at the single cell level, showing no consistent patterns (Figure $2 \mathrm{G}$ ). These results are consistent with recent single cell Hi-C measurements (Nagano et al., 2013; Stevens et al., 2017). We note that intron seqFISH provides complementary information about the spatial organization of the nucleus compared to ensemble Hi-C (Lieberman-Aiden et al., 2009; Dixon et al., 2012), which captures features, such as topological associated domains, that are highly consistent amongst cells. As TAS are randomly distributed at chromosomal interfaces, their spatial organizations are averaged out in ensemble experiments.

\section{Transcriptome level intron seqFISH, mRNA, IncRNA seqFISH and immunofluorescence in single cells.}

We next sought to characterize the instantaneous transcriptional activity further at the transcriptome level as well as its relationship with the transcriptional states of the cell and nuclear bodies. To extend intron seqFISH to the transcriptome level, we designed probes targeted 10,421 genes and decoded these genes with 8 rounds of hybridization $\left(4^{8}=65,536\right.$; Figure $\left.3 A-B\right)$, which includes one round of error correction. We followed the intron seqFISH experiment with several addition rounds of mRNA, IncRNA seqFISH and antibody staining to target pluripotency, differentiation and cell cycle markers in addition to nuclear bodies in the same single cells (Figure 3A-B). We observed that $51 \pm 29 \%$ (mean \pm standard deviation) of the dots detected in the first round of hybridization are decoded per cell, with the lower rate of detection due to the fewer number of probes ( 24 per gene), limited by cost (a total of 250,104 probes were synthesized for all 10,421 genes), as well as loss of signal and alignment over 8 rounds of hybridization. The false positive rate for the 10,421 intron seqFISH experiment is estimated at $2.1 \pm$ $1.2 \%$ per cell. We observed uniform spatial distribution of TAS in mESC nuclei (Figure $3 C$ ), consistent with the 1,000 gene intron seqFISH results (Figure $2 \mathrm{~A}$ ).

Surprisingly, large variabilities in the overall instantaneous transcriptional states of cells are present, as determined by the number of active transcription sites in each nucleus in the 10,421 gene intron experiment (Figure 3D). There are on average $990 \pm 634$ TAS per cell (mean \pm standard deviation), indicating that some cells are globally transcriptional active while other cells are globally quiescent. Similarly, wide distributions are also observed in the 1000 gene intron experiments (Figure 3D). Notably, the variability in TAS number still exists within same cell cycle phases, which are distinguished based on immunofluorescence. Nuclear volume, on the other hand, is correlated with the total TAS numbers, but significant variations remain in each cell size window. Therefore, even when cell cycle and size variabilities are taken into account, there are substantial variabilities in global instantaneous transcriptional levels among single cells.

Using the IncRNA and immunofluorescence staining, we examined the spatial relationship between the TAS and nuclear bodies in mESCs. We observed that TAS are not strongly colocalized with nuclear bodies, such as the paraspeckle marked by Neat1, nuclear speckle marked by Malat1 and SC35, and IncRNA Firre, which controls a network of genes related to RNA processing in mESCs (Bergmann et al., 2015) (Figure 4A-B). Interestingly, while probes targeting polyA sequences are colocalized with SC35 speckles (Figure 4B and 4D), intron probes targeting TAS are weakly colocalized with SC35. We also observed heterogeneous expression of Malat1 and intermediate co-localization between Malat1 and SC35 labeled nuclear speckles. These observations are consistent with literature (Politz et al., 2006), and 
suggest that active transcription sites are not necessarily recruited to nuclear speckles.

\section{Global dynamics in transcriptional activities}

The large variability in global transcriptional states raises the question of whether these global states are static in time or interconvert dynamically. Based on the weak correlation $(R=0.28)$ between the total TAS number in the nucleus and the amount of mature mRNA in the cytoplasm measured by dT oligos (Figure 4C), we hypothesized that the nascent global activities are not static in time. As the dynamics are unlikely to be synchronized amongst cells, we cannot measure the interconversion rate between active and inactive global transcriptional states by population averaged experiments. At the same time, it is also difficult to perform direct live cell experiments with reporter based assays to measure the transcriptional activities across many genes.

To overcome these limitations and measure the dynamics of TAS globally, we developed a single cell pulse chase experiment that records the nascent transcriptional activities at two time points in a cell's history (Figure 5A). We first fed cells with a modified Uridine (5-EU) to record the global transcriptional activity during a short 30 minutes pulse. Then we washed out the 5-EU and let the cells grow for different amounts of time from 0 to 2 hours. We fixed the cells, measured the 5-EU incorporation levels with a clickable fluorescent dye and counted the number of TAS with the 1000 gene intron probes in the same cells. The variability in the 5 -EU signal in individual cells is similar to the intron variability observed (Figure 3D), confirming nascent transcriptional heterogeneity in single cells.

Next, we determined whether transcriptional activities are changing or static over time by comparing the global instantaneous transcriptional activity at defined time points in the past labeled by 5-EU incorporation with the activity at the present measured by intron levels in the same cells (Figure 5B). At early time points, the 5-EU and intron levels are correlated in single cells (Figure 5B, top panel), confirming that the heterogeneities observed in both measurements are consistent. The correlation coefficient decayed within 1 hours, with little correspondence between the 5-EU signal and intron levels in single cells (Figure 5B, middle panel). This indicates that the overall nascent activity in cells are highly dynamic, with the global activity decorrelating within a one hour time period. Surprisingly, the correlation is restored at around 2 hours (Figure 5B, lower panel, and $5 \mathrm{C}$ ). This result suggests the transition of $\mathrm{mESCs}$ between low and high transcriptional states occurs with a 2 hour time period. Our data at each time point consist of hundreds of cells and the 2 hour oscillation is observed in multiple biological replicates as well as in a completely different line of mESCs (Figure 5C, right). It is also worth noting that the mean number of TAS per cell did not vary between pulse-chase time points, and only the correlations with the 5-EU levels at the single cell level oscillated.

This fast dynamics in the global nascent transcription is abolished in $2 \mathrm{i}$ condition (Figure 5C, red lines). It has previously been shown that mESCs in $2 \mathrm{i}$ conditions are globally hypomethylated and have homogeneous expression levels of pluripotency factors such as Rex1, Esrrb and Nanog (Singer et al., 2014). Our observations indicate that the mESCs in the naïve state in $2 \mathrm{i}$ conditions are static in time and that oscillation is an intrinsic feature of mESCs in the metastable pluripotent state in serum media where significant heterogeneity in transcriptional and epigenetic levels are observed (Marks et al., 2012; Singer et al., 2014; Kolodziejczyk et al., 2015).

Taken together, our finding of the global instantaneous transcriptional level fluctuations at the fast time scale is consistent with the lack of correlation with the mature mRNA levels in single cells (Figure 4C), as 
the longer lifetimes of mRNAs average out the fast dynamics in nascent transcriptional activities. As seen in a high degree of heterogeneity of the instantaneous transcriptional activities at any given time point, mESCs in the culture are not synchronized. However, cells interconvert between high and low instantaneous transcriptional states with a constant period of oscillation. If the global transcriptional activities were fluctuating stochastically without a defined period, or if different cells had different oscillation periods, then the correlation coefficients would simply decay without re-cohering at 2 hours.

\section{Discussion.}

In this study, we perform highly multiplexed intron seqFISH to capture the instantaneous activity of the transcriptome in large numbers of single mESCs. We combine these measurements with multiple rounds of mRNA seqFISH, IncRNA seqFISH and immunofluorescence to characterize transcriptional states, nuclear bodies and cell-cycle phases in the same single cells. This single-cell highly multiplexed imaging approach is complementary to sequencing-based methods such as GRO-seq (Core et al., 2008; Jonkers et al., 2014) and Hi-C (Lieberman-Aiden et al., 2009; Dixon et al., 2012) in measuring the nascent transcriptional activity and chromosome organization, respectively. Even though the seqFISH approach does not have nucleotide level resolution on chromosomal loci, imaging allows direct mapping of spatial organization beyond pairwise interactions measured in $\mathrm{Hi}-\mathrm{C}$ and provide spatial context with respect to nuclear bodies through immunofluorescence and IncRNA staining in the same cells. In addition, the single molecule based seqFISH methods allows sensitive detection of nascent transcripts in single cells, currently not available with sequencing methods. Lastly, combining intron FISH with pulse labeling provides dynamic information that would be otherwise lost in population average measurements or in reporter-based single gene live cell experiments.

The spatial genomics approach provides a systematic view of the organization of nascent transcription active sites in mESCs (Figure 6A). Specifically, we found that first, TAS are in general distributed at the surface of chromosome territories, with some loci looped away from the CTs, and spread across the nucleus with the exclusion of nucleoli and heterochromatic regions. Second, TAS in different chromosomes can be located in close proximity and these inter-chromosomal contacts are highly variable among single cells, consistent with reports that regions where chromosomes intermingle are enriched with transcriptionally active phosphorylated RNAPII in mESCs (Maharana et al., 2016). Third, instantaneous transcriptional activity does not affect spatial positioning of the genomic loci relative to their CTs, and those loci appear at the surface of CTs even they are transcriptionally quiescent. This is consistent with previous reports that instantaneous transcriptional differences may not affect chromosome positioning from nuclear periphery (Levesque and Raj, 2013) and between homologous alleles (Takebayashi et al., 2012). However, it is possible that changes of transcriptional activity could drive reorganization of chromosome conformation at specific cases or regions such as active and inactive X chromosomes (Naughton et al., 2010), and HoxB cluster (Chambeyron and Bickmore, 2004). Further investigation on these specific loci can take advantage of intron seqFISH together with multiplexed live cell imaging of genomic loci (Takei et al., 2017).

Our data further showed that instantaneous global transcriptional activities have large variabilities amongst cells. These global states oscillate on the time scale of 2 hours in mESCs cultured under metastable serum + LIF conditions (Figure 6B) but not in naïve $2 \mathrm{i}$ conditions, where mESCs are known to be less heterogeneous and have reduced DNA methylation levels (Singer et al., 2014). Indeed, a recent study discovered that DNA methylation also oscillates with 2 hour periods in mESCs released from naïve 
into primed culture conditions (Reik, personal communication). Furthermore, it has reported that Hes1, DII1, and Gadd45g promoter activities oscillated on a 2-5 hour time scale in mESCs (Kobayashi et al., 2009). These observations suggest that global oscillations in both DNA methylation and transcriptional activity may be connected and should be further explored.

The unexpected dynamics in the global transcription activities in mESCs shows that single cell whole transcriptome profiling and pulse chase experiments can reveal hitherto unknown regulatory dynamics. Using dynamic regulatory mechanisms, cells can achieve states not accessible with amplitude based regulation schemes (Letsou and Cai, 2016). For example, cells can use fluctuations in global transcriptional activity to coordinate the stoichiometry of many transcripts in a mechanisms akin to the frequency modulated signaling observed in yeast and mammalian pathways (Cai et al., 2008; Yissachar et al., 2013). It will be fascinating to determine the mechanisms underlying this oscillation and investigate whether similar fast dynamics occur in the inner cell mass of embryos. 

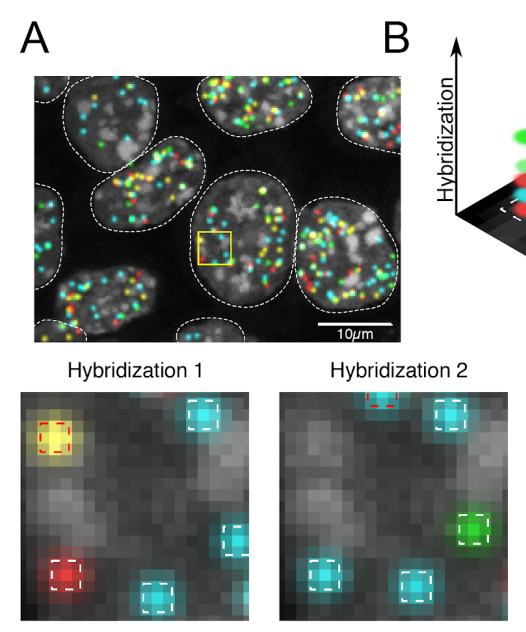

Barcode Composite
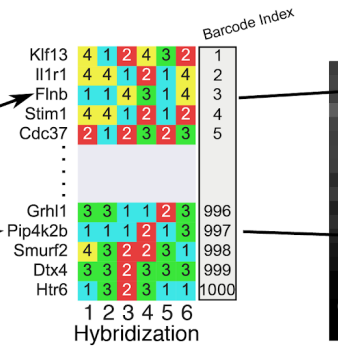

Gene Assignment
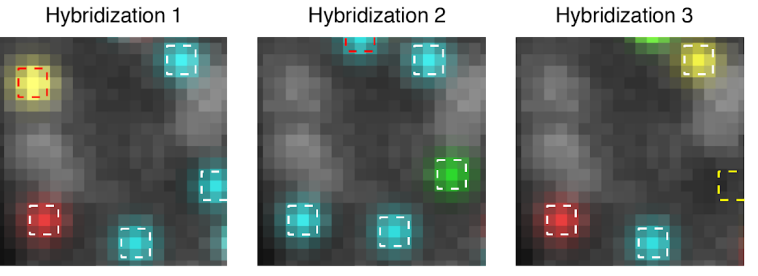

Hybridization 4
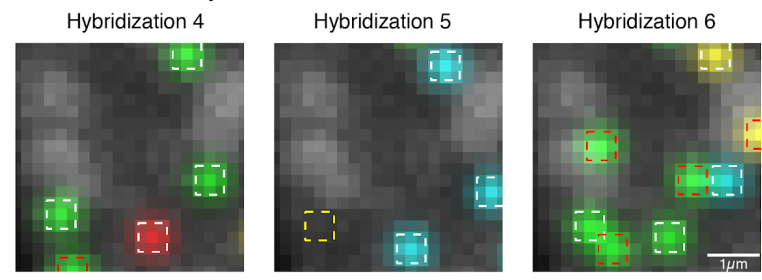

C

D
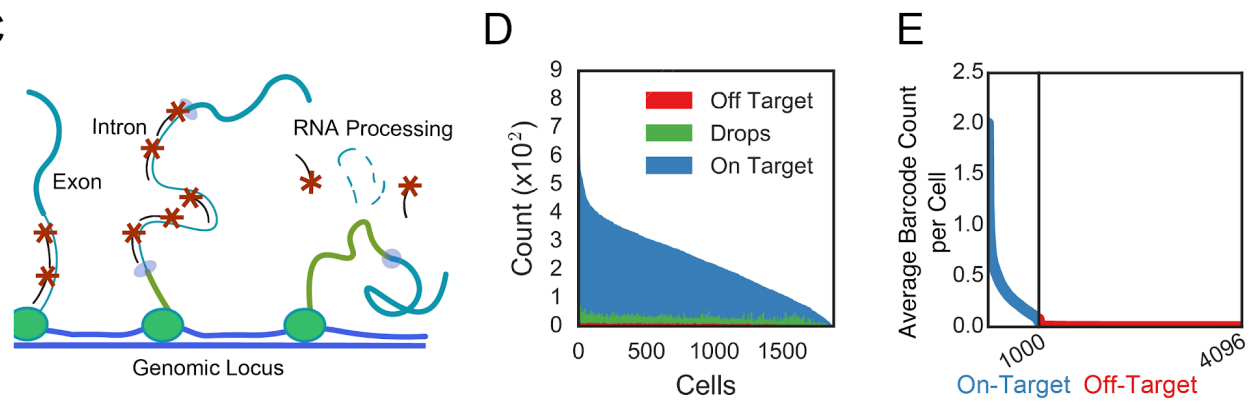

Figure 1. Intron seqFISH enables profiling of active transcription. (A) Large field of view image of multiple $\mathrm{mESC}$ in a single hybridization from a single z-section. Below is a zoomed-in view of the boxed region over 6 sequential hybridizations. White boxes indicate identified barcodes, yellow boxes are recovered signal from error corrected barcodes, red boxes indicate false positive spurious hybridizations. (B) Identification of barcodes from zoomed region in A. The entire detected barcode is depicted as a concatenation of all 6 hybridizations. The genomic position of nascent RNA molecules is mapped by looking up barcode identities in a pre-assigned table. (C) Schematic depiction of intron FISH. Many nascent RNA molecules are present at the transcription active site, amplifying the detected signal. (D) Several hundred introns are typically detected per cell. Few off-target or incomplete barcodes are detected in any cell. (E) Average number of real (on-target) and false positives (off-target) introns detected per cell. Introns display a wide range of expression patterns, from near bi-allelic expression in every cell, to rare expression. False (off-target) introns are rarely detected, demonstrating the accuracy of intron seqFISH. 


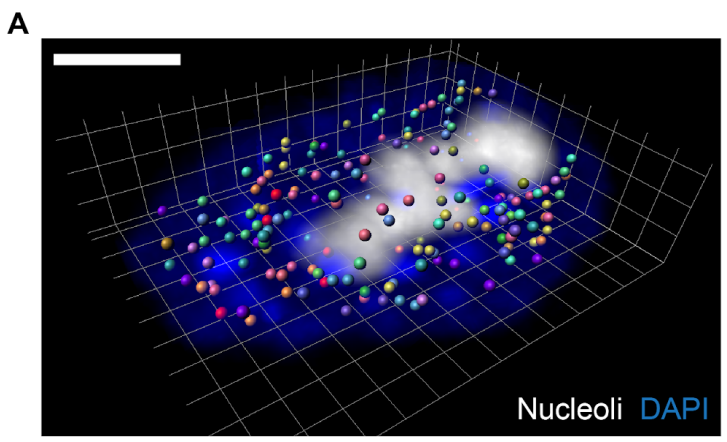

Chr 1-19,X

B

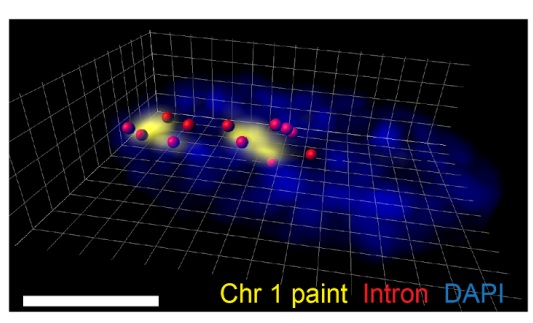

D
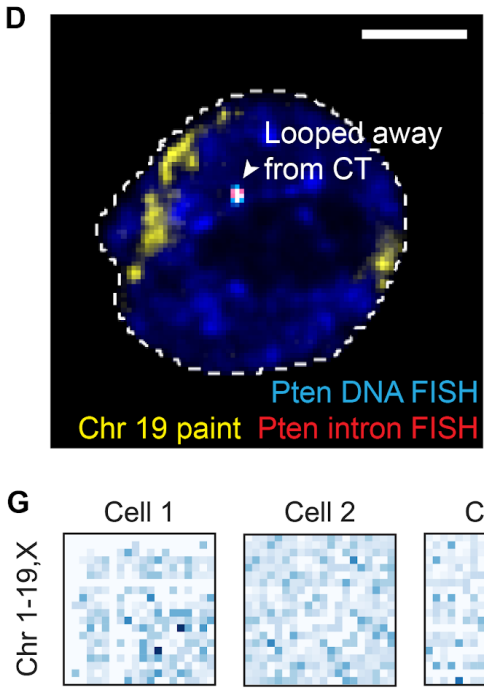

Chr 1-19,X

E

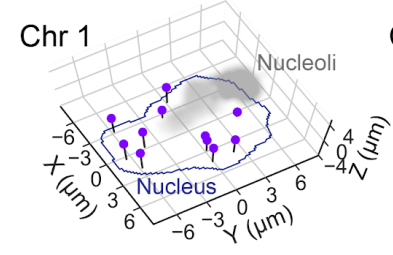

Chr 2

Chr 15

Chr X
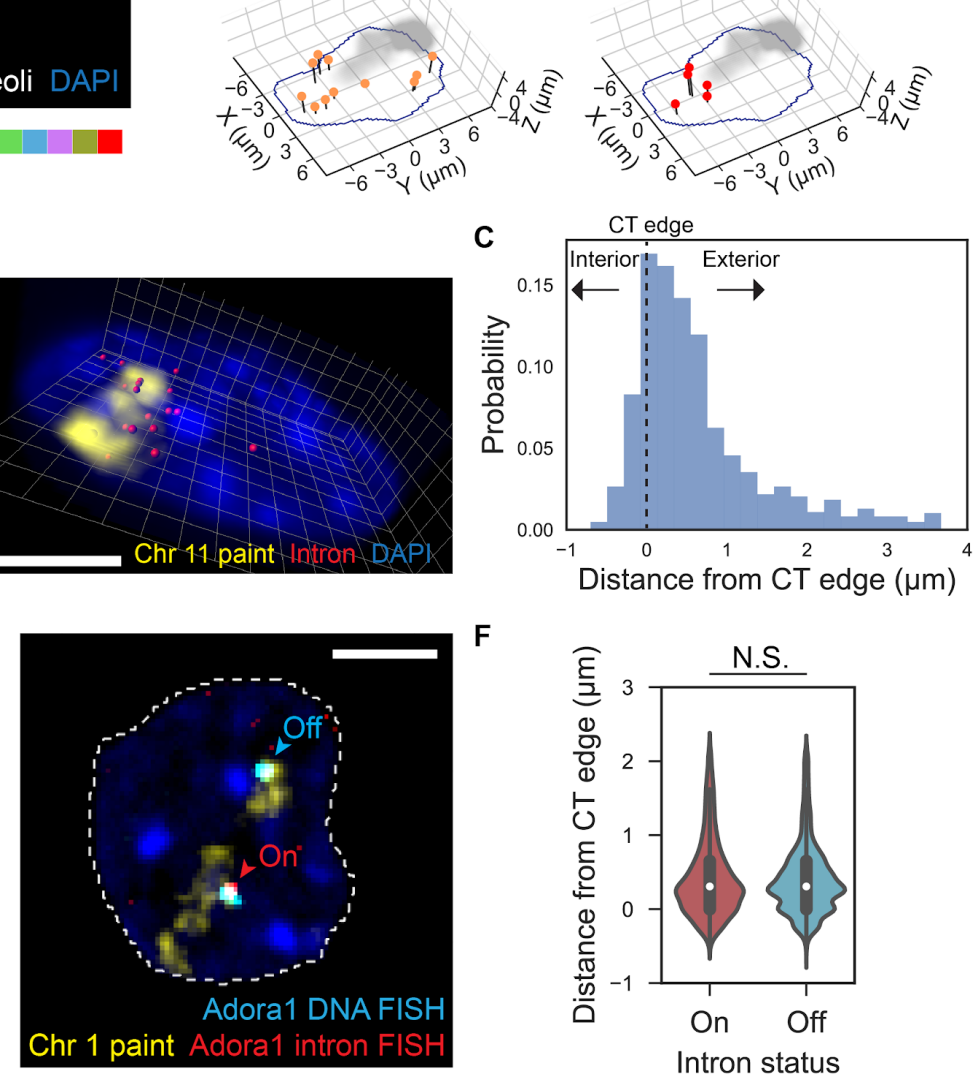
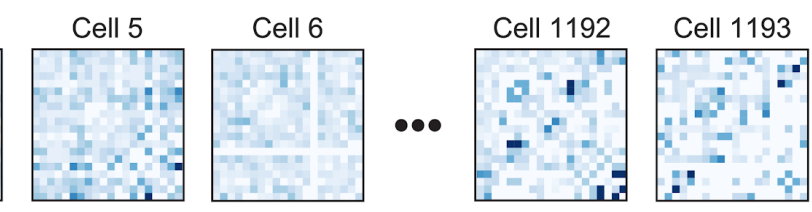

Normalized contact frequency

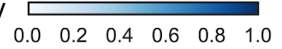

Figure 2. The 1,000 intron seqFISH reveals nascent transcription active sites at the surface of chromosome territories. (A) 3D reconstruction of TAS in a single mESC nucleus, with individual chromosome occupying distinct spatial territories (right). In total, 180 nascent sites were present in this cell. Nucleoli were labeled by ITS1 FISH and nucleus was stained by DAPI. (B) 3D reconstruction of introns from a particular chromosome (red) and chromosome paint of the same chromosome (yellow) in mESC nuclei (blue), showing nascent active sites present on the surfaces of chromosome territories. Chromosome 1 (left) and chromosome 11 (right) (intron probes targeting 77 and 79 genes on their chromosomes) are shown respectively. (C) Histogram showing the distance distribution of TAS relative to their chromosomal territory (CT) edge in $\mathrm{mESC}$ nuclei ( $n=1472$ spots from 4 chromosomes in total). 
(D) Locus looped away from its core CT, shown by DNA FISH (cyan) and intron FISH (red) targeting Pten along with chromosome 19 paint (yellow) in a mESC nucleus (blue). (E) Transcriptional status of loci does not affect their spatial positioning with respect to the CT boundary. DNA FISH (cyan) and intron FISH (red) targeted both allele of a gene (Adora1, as an example) along with chromosome 1 paint (yellow) in a mESC nucleus (blue). Signals outside nuclei (dashed white lines) are not shown for visual clarity (D, E). (F) Violin plots showing the distance distribution relative to its CT edge for loci with either "on" or "off" intron signals. N.S., not significant with Wilcoxon's rank sum test $(P>0.05)$. Results from 9 genes in chromosome 1, including Adora1, are shown ( $n=1550$ and 4298 spots for "on" and "off" status). Scale bars (A, B, D, E), $5 \mu \mathrm{m}$. (G) Heat maps representing normalized contact frequency of TAS between pairs of chromosomes in single nuclei. 
A

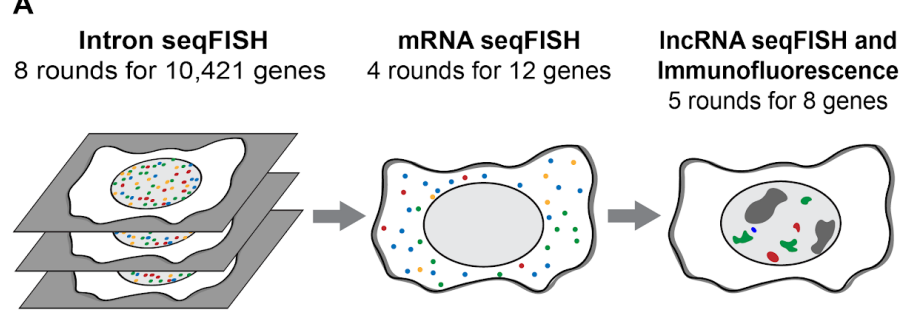

C

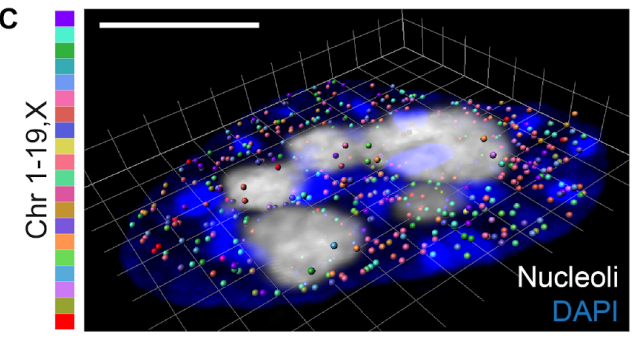

\section{B}
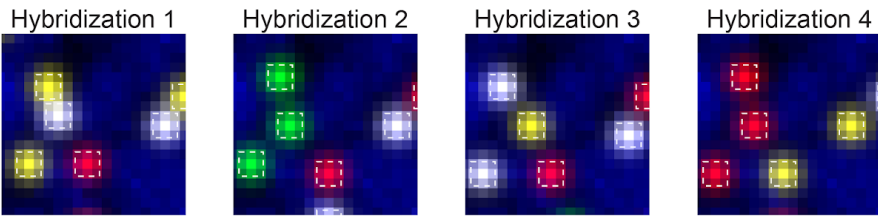

Hybridization 5

10,421 gene intron seqFISH

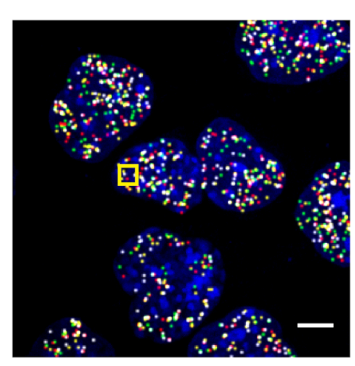

Hybridization 6

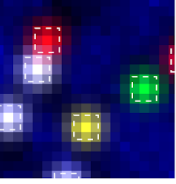

Hybridization 7
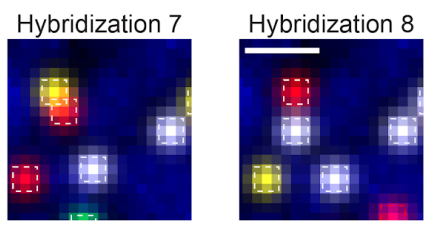

Gene assignment

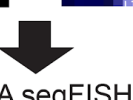

mRNA seqFISH

IncRNA seqFISH

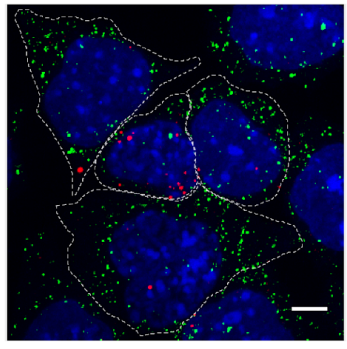

Krt8 Sox2 DAPI

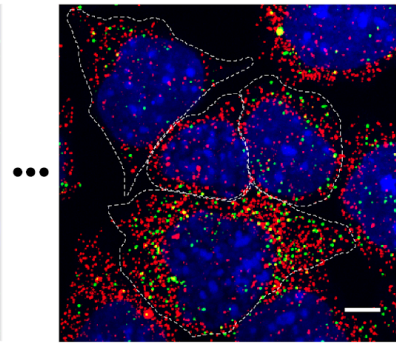

Oct4 Aurka DAPI

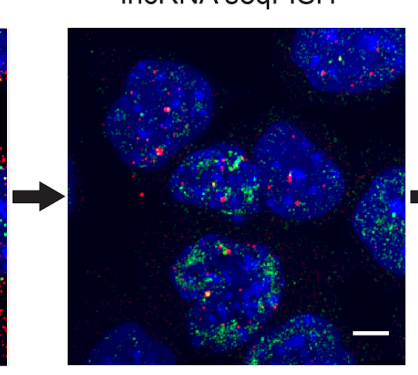

Neat1 Malat1 DAPI
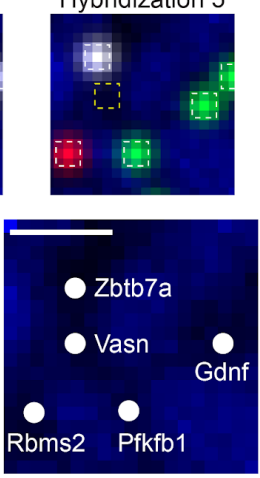

Immunofluorescence

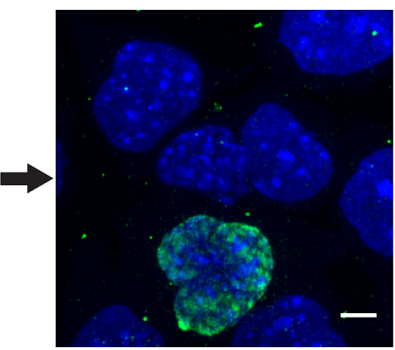

H3S10Ph DAPI

D

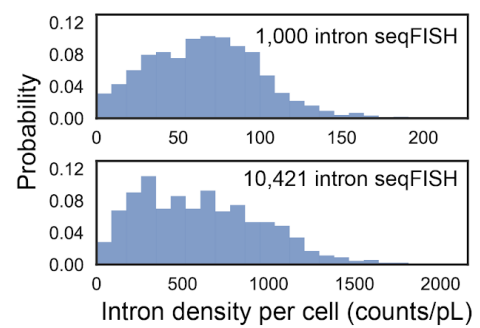

Figure 3. Transcriptome level seqFISH reveals heterogeneity in global nascent transcription. (A)

Schematic of the combined seqFISH and immunofluorescence single cell measurements. 10,421 intron seqFISH decoded by 8 rounds of multiplexed barcoding were followed by additional rounds of mRNA, IncRNA seqFISH and immunofluorescence. (B) Combined seqFISH and immunofluorescence images with multiple mESCs. Barcodes in the boxed region of 10,421 gene intron seqFISH image are magnified. Colored boxes are depicted in the same way as Figure 1A. Note that intron barcodes are combined from 3 z-slices and digitized for visualization. Other images are maximum intensity projections of a z-stack of fluorescence images. Dashed white lines in mRNA seqFISH images display cytoplasmic boundaries of cells. Scale bars represent $5 \mu \mathrm{m}$ in images with multiple single cells, and $1 \mu \mathrm{m}$ in the magnified images. (C) 3D reconstruction of TAS from 20 chromosomes (chr1-19, X) in a single mESC nucleus. In total, 573 
bioRxiv preprint doi: https://doi.org/10.1101/339234; this version posted June 7, 2018. The copyright holder for this preprint (which was not certified by peer review) is the author/funder, who has granted bioRxiv a license to display the preprint in perpetuity. It is made available under aCC-BY-NC-ND 4.0 International license.

nascent sites were decoded in this cell by the 10,421 gene intron seqFISH. Nucleoli were labeled by ITS1 FISH and nucleus was stained by DAPI. Scale bar represents $5 \mu \mathrm{m}$. (D) Histograms showing wide distributions of nascent site density per cell from the 1000 gene intron seqFISH (top) and 10,421 gene intron seqFISH (bottom) ( $\mathrm{n}=1193$ and 885 cells). Counts are normalized by nuclear volume ( $\mathrm{pL}$, picoliter). 


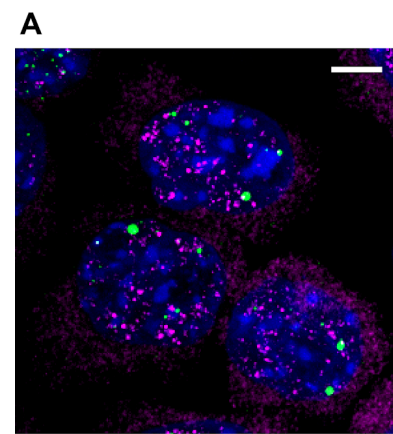

Intron Neat1 DAPI

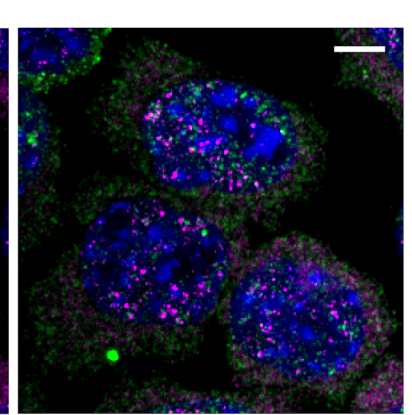

Intron Malat1 DAPI

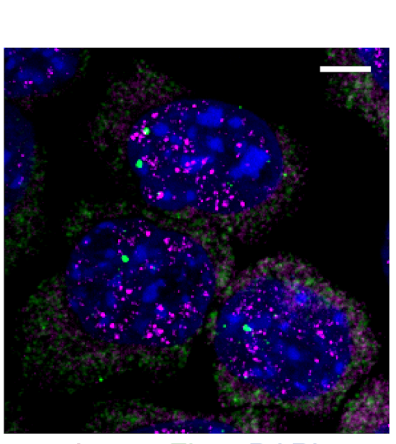

Intron Firre DAPI

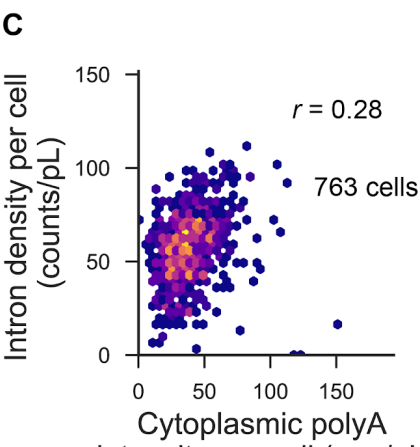

intensity per cell (a.u./pL)
B

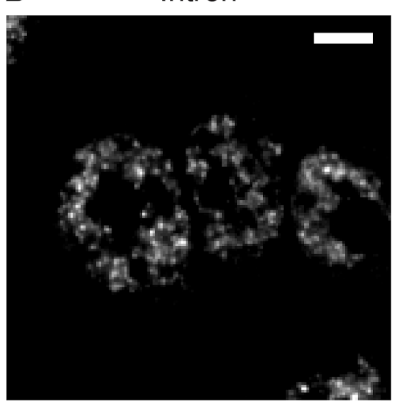

SC35

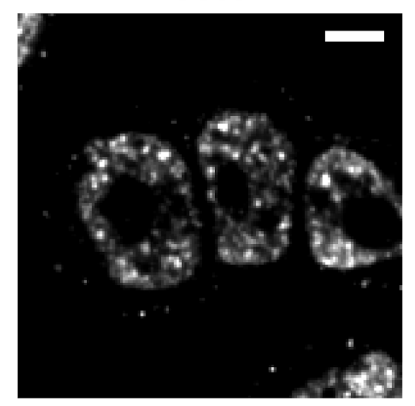

PolyA

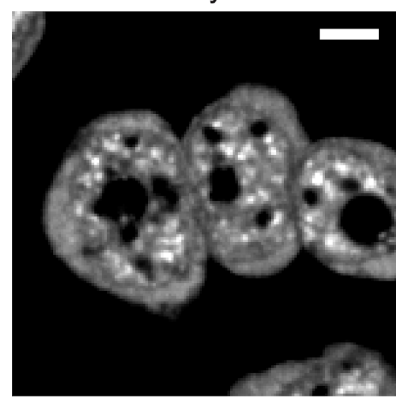

DAPI

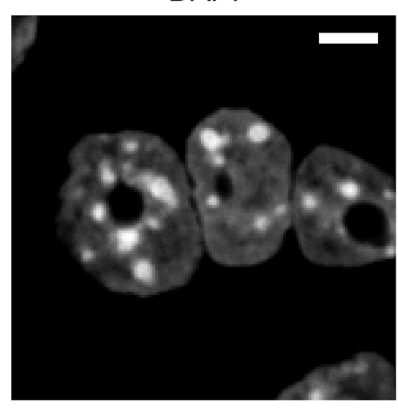

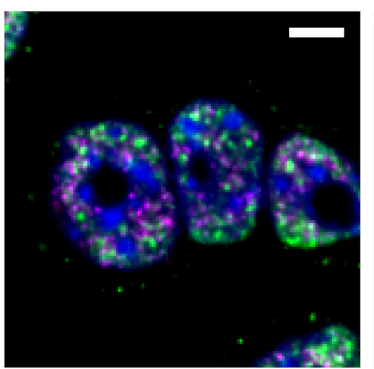

Intron SC35 DAPI

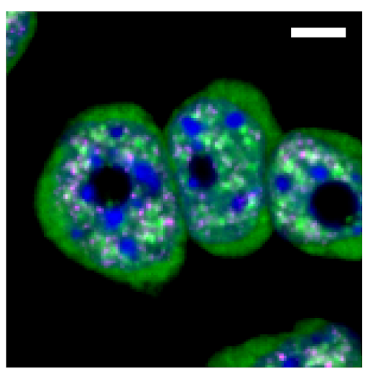

Intron PolyA DAPI

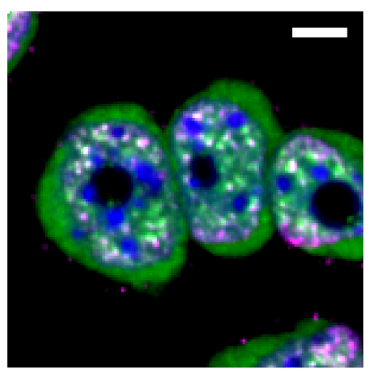

SC35 PolyA DAPI

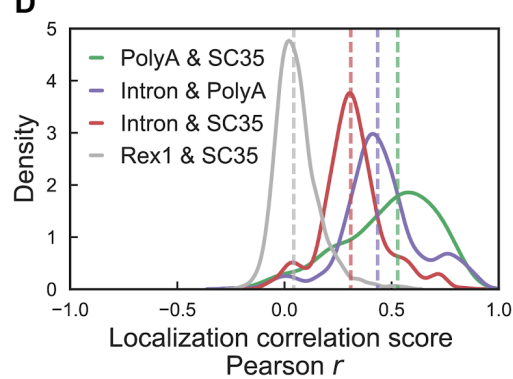

Figure 4. Organization of transcription active sites and nuclear bodies. (A) Representative images showing intron spots from the Alexa 488 channel in the first hybridization of the 10,421 gene intron seqFISH (magenta), IncRNAs by IncRNA seqFISH (green) and nuclear stain by DAPI (blue) in mESCs. Images are maximum intensity projections of a z-stack of fluorescence images. (B) Representative images showing intron spots from 1000 gene seqFISH, polyA FISH, SC35 immunofluorescence, and nuclear stain by DAPI. (C) Pairwise relationship between intron density by the 1000 gene intron seqFISH and cytoplasmic polyA intensity by polyA FISH ( $n=763$ cells). $r$, Pearson correlation coefficient. (D) Distributions of localization correlation scores (Pearson correlation coefficient) in single cells ( $n=437$ nuclei). Solid lines display density plots and dashed lines indicate median correlation scores from our data. Note that Rex1 (mRNA FISH) \& SC35 correlation score represents baseline correlation. Scale bars (A, B), $5 \mu \mathrm{m}$. 
A

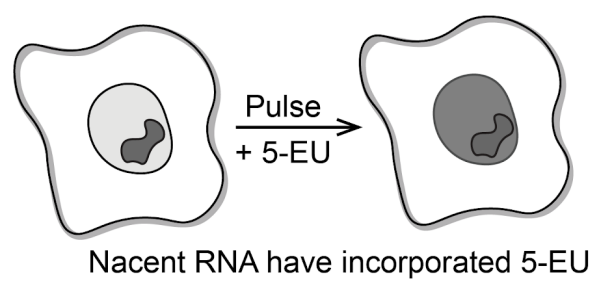

C

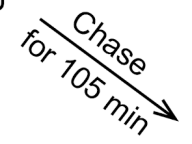

E14 cells

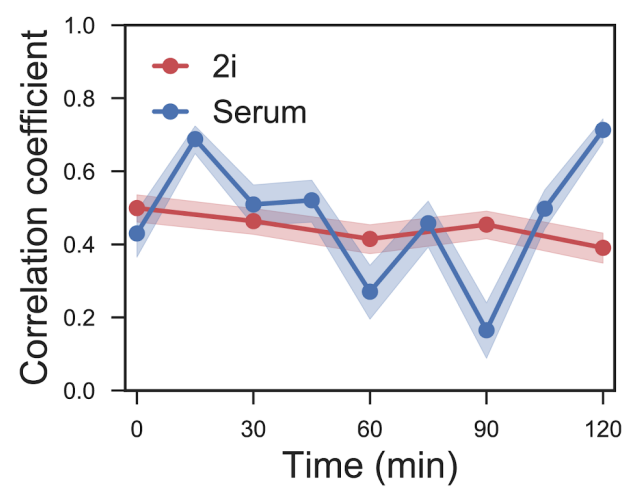

B
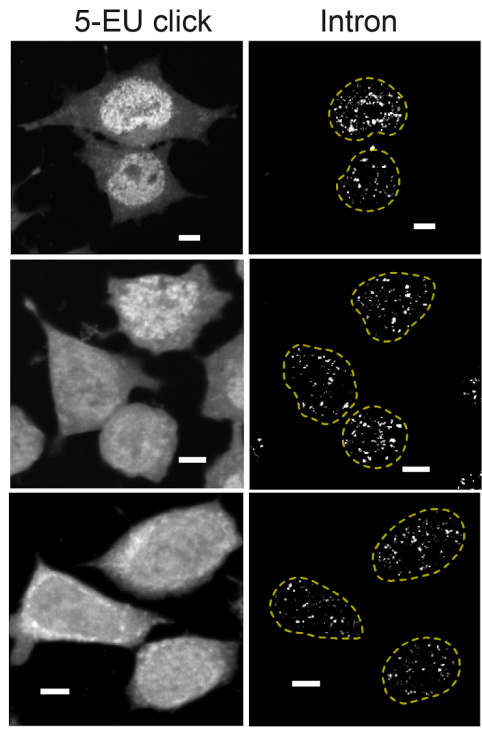

Wild-type mESCs

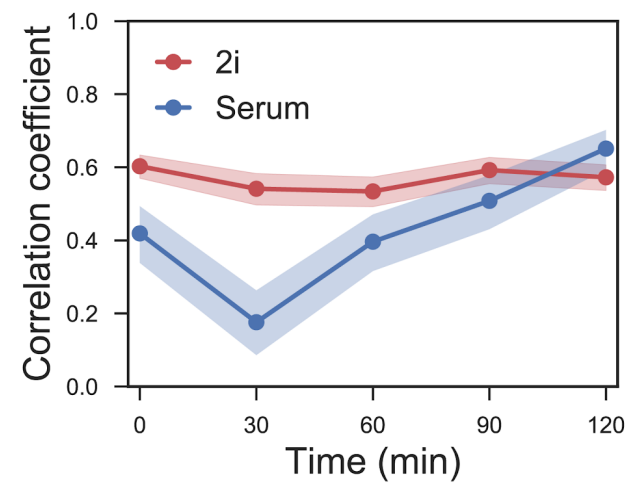

Figure 5. Single cell pulse chase experiments reveal fast dynamics in global nascent transcription. (A) Schematic of the pulse-chase experiment. 5-EU was pulsed for $30 \mathrm{~min}$ to globally label nascent transcripts, then chased for different periods of time in growth medium lacking 5-EU, followed by fixation. (B) Images of 5-EU detected by Click linkage to an azide-dye, and 1000 gene introns in single cells. The correlations of 5-EU and intron examined in different chase time were shown as scatterplots with Pearson correlation coefficient $(r)$, for $0 \mathrm{~min}, 30 \mathrm{~min}$, and $105 \mathrm{~min}$ chase periods, respectively. $(\mathrm{n}=$ 1495, 780 and 1176 cells). Dashed yellow lines display nuclear boundaries determined by DAPI. Scale bars, $5 \mu \mathrm{m}$. (C) Pulse-chase correlation measurements show oscillatory dynamics on the time scale of two hours. Oscillations are observed in two cell lines of mESCs (left and right panels). In contrast to cells in serum conditions (blue), cells in $2 \mathrm{i}$ conditions do not show oscillations (red). Shaded regions represent $95 \%$ confidence intervals. 
A

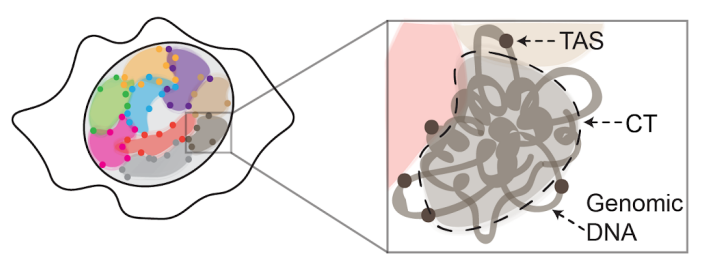

B

Cell 1

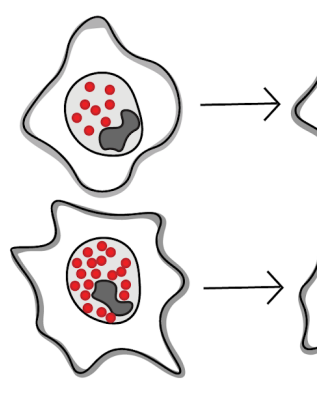

$0 \mathrm{hr}$

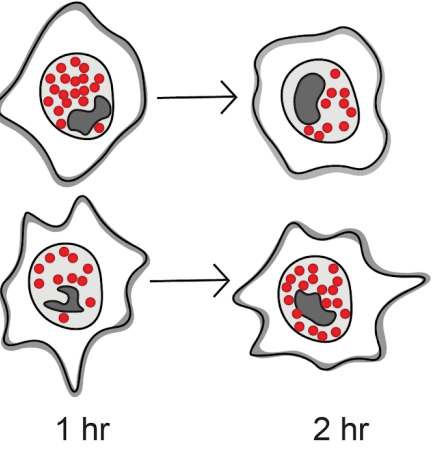

Figure 6. A model for spatial organization and dynamics of global nascent transcription in mESCs. (A) transcription active sites (TAS) are organized at the surface of chromosomal territories (CTs) and interfaces. (B) Oscillatory dynamics in global nascent transcription activity in two different cells. Red dots indicate TAS. Dark patches represent nucleoli. 


\section{REFERENCES}

Angermueller $\mathrm{C}$, Clark SJ, Lee $\mathrm{HJ}$, et al. Parallel single-cell sequencing links transcriptional and epigenetic heterogeneity. Nature methods. 2016;13(3):229-232. doi:10.1038/nmeth.3728.

B. J. Beliveau et al., Versatile design and synthesis platform for visualizing genomes with Oligopaint FISH probes. Proceedings of the National Academy of Sciences of the United States of America 109, 2130121306 (2012).

Bergmann JH, Li J, Eckersley-Maslin MA, Rigo F, Freier SM, Spector DL. Regulation of the ESC transcriptome by nuclear long noncoding RNAs. Genome Res. 2015 Sep;25(9):1336-46.

Bolzer A, Kreth G, Solovei I, Koehler D, Saracoglu K, Fauth C, Müller S, Eils R, Cremer C, Speicher MR, Cremer T. Three-dimensional maps of all chromosomes in human male fibroblast nuclei and prometaphase rosettes. PLoS Biol. 2005 May;3(5):e157

S. Boyle, M. J. Rodesch, H. A. Halvensleben, J. A. Jeddeloh, W. A. Bickmore, Fluorescence in situ hybridization with high-complexity repeat-free oligonucleotide probes generated by massively parallel synthesis. Chromosome Res 19, 901-909 (2011).

M. N. Cabili et al., Localization and abundance analysis of human IncRNAs at single-cell and singlemolecule resolution. Genome Biol 16, 20 (2015).

L. Cai, C. K. Dalal, M. B. Elowitz, Frequency-modulated nuclear localization bursts coordinate gene regulation. Nature 455, 485-490 (2008).

I. Chambers et al., Nanog safeguards pluripotency and mediates germline development. Nature 450, 1230-1234 (2007).

Chambeyron S, Bickmore WA. Chromatin decondensation and nuclear reorganization of the HoxB locus upon induction of transcription. Genes Dev. 2004 May 15;18(10):1119-30.

Core $\mathrm{L}$, Waterfall JJ, Lis JT. Nascent RNA sequencing reveals widespread pausing and divergent initiation at human promoters. Science. 2008 Dec 19;322(5909):1845-8.

J. R. Dixon et al., Topological domains in mammalian genomes identified by analysis of chromatin interactions. Nature 485, 376-380 (2012).

D. A. Faddah et al., Single-cell analysis reveals that expression of nanog is biallelic and equally variable as that of other pluripotency factors in mouse ESCs. Cell Stem Cell 13, 23-29 (2013).

Fulton DL, Sundararajan S, Badis G, Hughes TR, Wasserman WW, Roach JC, Sladek R. TFCat: the curated catalog of mouse and human transcription factors. Genome Biol. 2009;10(3):R29. doi: 10.1186/gb-200910-3-r29.

K. Hayashi, S. M. Lopes, F. Tang, M. A. Surani, Dynamic equilibrium and heterogeneity of mouse pluripotent stem cells with distinct functional and epigenetic states. Cell Stem Cell 3, 391-401 (2008). 
Hu X, Zhang L, Mao SQ, Li Z, Chen J, Zhang RR, Wu HP, Gao J, Guo F, Liu W, Xu GF, Dai HQ, Shi YG, Li X, Hu B, Tang F, Pei D, Xu GL. Tet and TDG mediate DNA demethylation essential for mesenchymal-toepithelial transition in somatic cell reprogramming. Cell Stem Cell. 2014 Apr 3;14(4):512-22.

Jao CY, Salic A. Exploring RNA transcription and turnover in vivo by using click chemistry. Proceedings of the National Academy of Sciences of the United States of America. 2008;105(41):15779-15784. doi:10.1073/pnas.0808480105.

Jonkers I, Kwak H, Lis JT. Genome-wide dynamics of Pol II elongation and its interplay with promoter proximal pausing, chromatin, and exons. Elife. 2014 Apr 29;3:e02407. doi: 10.7554/eLife.02407.

A. M. Klein et al., Droplet barcoding for single-cell transcriptomics applied to embryonic stem cells. Cell 161, 1187-1201 (2015).

Kobayashi T, Mizuno H, Imayoshi I, Furusawa C, Shirahige K, Kageyama R. The cyclic gene Hes1 contributes to diverse differentiation responses of embryonic stem cells. Genes Dev. 2009 Aug $15 ; 23(16): 1870-5$.

A. A. Kolodziejczyk et al., Single Cell RNA-Sequencing of Pluripotent States Unlocks Modular Transcriptional Variation. Cell Stem Cell 17, 471-485 (2015).

R. M. Kumar et al., Deconstructing transcriptional heterogeneity in pluripotent stem cells. Nature 516, 56-61 (2014).

Letsou W, Cai L. Noncommutative Biology: Sequential Regulation of Complex Networks. PLoS Comput Biol. 2016 Aug 25;12(8):e1005089.

M. J. Levesque, A. Raj, Single-chromosome transcriptional profiling reveals chromosomal gene expression regulation. Nat Methods 10, 246-248 (2013).

Lieberman-Aiden E, van Berkum NL, Williams L, Imakaev M, Ragoczy T, Telling A, Amit I, Lajoie BR, Sabo PJ, Dorschner MO, Sandstrom R, Bernstein B, Bender MA, Groudine M, Gnirke A, Stamatoyannopoulos J, Mirny LA, Lander ES, Dekker J. Comprehensive mapping of long-range interactions reveals folding principles of the human genome. Science. 2009 Oct 9;326(5950):289-93.

E. Lubeck, A. F. Coskun, T. Zhiyentayev, M. Ahmad, L. Cai, Single-cell in situ RNA profiling by sequential hybridization. Nat Methods 11, 360-361 (2014).

Maharana S, Iyer KV, Jain N, Nagarajan M, Wang Y, Shivashankar GV. Chromosome intermingling-the physical basis of chromosome organization in differentiated cells. Nucleic Acids Res. 2016 Jun 20;44(11):5148-60.

Mahy NL, Perry PE, Gilchrist S, Baldock RA, Bickmore WA. Spatial organization of active and inactive genes and noncoding DNA within chromosome territories. J Cell Biol. 2002 May 13;157(4):579-89.

Mahy NL, Perry PE, Bickmore WA. Gene density and transcription influence the localization of chromatin outside of chromosome territories detectable by FISH. J Cell Biol. 2002 Dec 9;159(5):753-63. 
Marks H, Kalkan T, Menafra R, Denissov S, Jones K, Hofemeister H, Nichols J, Kranz A, Stewart AF, Smith A, Stunnenberg HG. The transcriptional and epigenomic foundations of ground state pluripotency. Cell. 2012 Apr 27;149(3):590-604.

Nagano T, Lubling Y, Stevens TJ, Schoenfelder S, Yaffe E, Dean W, Laue ED, Tanay A, Fraser P. Single-cell $\mathrm{Hi}-\mathrm{C}$ reveals cell-to-cell variability in chromosome structure. Nature. 2013 Oct 3;502(7469):59-64.

Naughton C, Sproul D, Hamilton C, Gilbert N. Analysis of active and inactive X chromosome architecture reveals the independent organization of $30 \mathrm{~nm}$ and large-scale chromatin structures. Mol Cell. $2010 \mathrm{Nov}$ 12;40(3):397-409.

Politz JC, Tuft RA, Prasanth KV, Baudendistel N, Fogarty KE, Lifshitz LM, Langowski J, Spector DL, Pederson T. Rapid, diffusional shuttling of poly(A) RNA between nuclear speckles and the nucleoplasm. Mol Biol Cell. 2006 Mar;17(3):1239-49.

A. Raj, C. S. Peskin, D. Tranchina, D. Y. Vargas, S. Tyagi, Stochastic mRNA synthesis in mammalian cells. PLoS Biol 4, e309 (2006).

J.-M. Rouillard, M. Zuker, E. Gulari, OligoArray 2.0: design of oligonucleotide probes for DNA microarrays using a thermodynamic approach. Nucleic Acids Research 31, 3057-3062 (2003).

S. Shah, E. Lubeck, W. Zhou, L. Cai, In Situ Transcription Profiling of Single Cells Reveals Spatial Organization of Cells in the Mouse Hippocampus. Neuron 92, 342-357 (2016).

Sharova LV, Sharov AA, Nedorezov T, Piao Y, Shaik N, Ko MS. Database for mRNA half-life of 19977 genes obtained by DNA microarray analysis of pluripotent and differentiating mouse embryonic stem cells. DNA Res. 2009 Feb;16(1):45-58. doi:10.1093/dnares/dsn030

K. V. Shishova, O. Zharskaya, O. Zatsepina, The Fate of the Nucleolus during Mitosis: Comparative Analysis of Localization of Some Forms of Pre-rRNA by Fluorescent in Situ Hybridization in NIH/3T3 Mouse Fibroblasts. Acta Naturae 3, 100-106 (2011).

Z. S. Singer et al., Dynamic heterogeneity and DNA methylation in embryonic stem cells. Mol Cell 55, 319-331 (2014).

S. A. Smallwood et al., Single-cell genome-wide bisulfite sequencing for assessing epigenetic heterogeneity. Nat Methods 11, 817-820 (2014).

T. J. Stevens et al., 3D structures of individual mammalian genomes studied by single-cell Hi-C. Nature 544, 59-64 (2017).

Takei Y, Shah S, Harvey S, Qi LS, Cai L. Multiplexed Dynamic Imaging of Genomic Loci by Combined CRISPR Imaging and DNA Sequential FISH. Biophys J. 2017 May 9;112(9):1773-1776.

Y. Toyooka, D. Shimosato, K. Murakami, K. Takahashi, H. Niwa, Identification and characterization of subpopulations in undifferentiated ES cell culture. Development 135, 909-918 (2008). 
bioRxiv preprint doi: https://doi.org/10.1101/339234; this version posted June 7, 2018. The copyright holder for this preprint (which was not certified by peer review) is the author/funder, who has granted bioRxiv a license to display the preprint in perpetuity. It is made available under aCC-BY-NC-ND 4.0 International license.

Yissachar N, Sharar Fischler T, Cohen AA, Reich-Zeliger S, Russ D, Shifrut E, Porat Z, Friedman N. Dynamic response diversity of NFAT isoforms in individual living cells. Mol Cell. 2013 Jan 24;49(2):322-

30. 